\title{
Time-Sensitive Satellite Internet Based on Uniform Content Labels
}

\author{
Jie Shen ${ }^{1,2, *,}$, Wenqi Dong ${ }^{1,2, \dagger}$, Jing Wang ${ }^{1, \dagger}$, Peng Zang ${ }^{1}$, \\ Xuewei Shi ${ }^{1}$ and Yang Wang ${ }^{1}$
}

\author{
${ }^{1}$ State Grid Jibei Zhangjiakou Wind and Solar Energy Storage and Transportation \\ New Energy Co., Ltd, China \\ ${ }^{2}$ Beijing University of Posts and Telecommunications, Beijing, China \\ E-mail: shenjie74@163.com \\ * Corresponding Author \\ ${ }^{\dagger}$ These authors contributed equally to this work and should be considered co-first \\ authors
}

Received 25 August 2021; Accepted 01 November 2021;

Publication 07 January 2022

\begin{abstract}
For satellite Internet the content was examined by location and time of day, as well as within the context of online social networks. The ground Information-Centric Networking (ICN) is a paradigm shift from host-to-host Internet Protocol (IP)-based communication to content-based communication. With the development and universal application of satellite technology, an important way to expand the function of satellites is setting up intersatellite networks to make them work together. The Internet and the Global Positioning System are based on the dual structure network and the Uniform Content Label UCL national standard. Through the integration of these two inventions, the integration of time and space is realized, creating an endogenous security environment of both mathematics and physics that is self-consistent in cyberspace, is expected to bring a breakthrough in principle for cracking network security challenges.
\end{abstract}

Keywords: Uniform content label, satellite internet, time-sensitive network.

Journal of Web Engineering, Vol. 21_2, 523-544.

doi: 10.13052/jwe1540-9589.21217

(C) 2022 River Publishers 


\section{Introduction}

Communication satellites for Internet communications are becoming more and more widespread. In addition to satellite communications covering the world, it can also conduct human-computer interactions in the Internet of Things. For example, the satellite Internet information transmission process for containers is generally as follows: firstly, the container information is collected [1,2]; secondly, the data is transmitted to the satellite through the satellite terminal; then the data is transmitted to the ground station; and finally, through the core network and the computer cloud computing platform for processing. The information is then transferred to the user via the reverse link. Among them, the current satellite terminals and ground stations can be designed together, that is, they are logically a set of systems. The terminal can also run the function of a ground station, that is, it can be regarded as a ground station.

The container information can be collected with sensors or electronic tags installed on it. This generalized tag can be considered as a Uniform Content Label (UCL). When there are more containers, labels will be used to indicate this information, instead of the current form of short messages. The actual ground network from the collector to the satellite terminal includes shortrange wired networks, wireless networks, and the Internet of Things, such as Lora, Bluetooth, $\mathrm{WiFi}$, and fibre optics.

The information to be transmitted: When to transmit and what kind of information is combined to transmit, it is determined by the satellite terminal, which can play the role of an edge computer. On the one hand, the information of the electronic label is stored locally, and on the other hand, how to transfer the information is determined by calculation. Satellite terminals are similar to mobile phones, small transceivers plus dish antennas, and terrestrial relay base stations, in many forms. At present, there are satellite terminals that take on the function of the ground station, that is, in the backhaul network, the data is not transmitted to the ground station, but to another terminal. Or a direct response communication between the satellite and the terminal.

The role of the satellite is similar to a wireless base station or switch on the ground. It can assume the function of label switching (MPLS) [4]. Studies have shown that the terrestrial MPLS protocol can be combined with satellite communications, and the Space Data Transmission Association (CCSDS) is also studying an improved IP protocol for use in satellites.

The satellite network is Time-Sensitive Networking (TSN), and IEEE's task group specifies a series of standards such as $802.1 \mathrm{Qbv}$ for enhancing the management of time-critical flows in real-time networks [5]. 
After the data is transmitted to the ground by satellite, it is collected by the ground station, and the computer system rearranges and combines the received data to decode the original information [6]. The computer system can also classify and count the received satellite data, which is the existing satellite space-time big data function. The ground computer network (or cloud platform) assumes the functions of the core network in the previous telecommunications network.

\section{Method of UCL on Space Internet}

In the satellite Internet, the content management challenges such as the obvious trend of content big data, the lack of content semantic identification and the severe content security situation, adopt the Uniform Content Label UCL national standard and rich semantic vector coding, combined with the protocol structure of spatial data transmission to form a source and channel integration, a space-time self-consistent dual-architecture satellite Internet and content intelligent management architecture. In order to solve the problem of content big data governance, the dual-architecture satellite Internet provides the self-certification and credibility of time-space structure on mathematics and physics, and provides innovative solutions at the network architecture level [1].

\subsection{Dual-architecture Internet Supporting of Cyber Content}

The dual-architecture satellite Internet follows the three design principles of the new Internet architecture, and innovates from three aspects: overall structure, core primitives, and governance methodology, and uses uniform content label (UCL, uniform content label) [7] content-driven primitives, Encode Internet content with rich semantic vector, establish a UCL multiidentity dimension semantic association model, introduce a security energy level model driven by data and knowledge, use knowledge graphs to describe semantic content associations, and establish a content big data UCL knowledge space.

UCL encodes content entities based on the five-element (5W) method, describing the five basic elements of when, where, who, what, and why. The principle of network architecture design is the "first problem" of computer network research, and the content-based links of satellite Internet also follow the three principles proposed by [8]. 
(1) Principle of dual-drive dual structure (P1). Satellite communication network is a new type of Internet with dual structure driven by content and address.

(2) The principle of rich semantic content primitives (P2). The content big data of the satellite network provides a unified format and rich semantic vectorized content identification.

(3) The principle of qualitative and quantitative integrated integration (P3). The principle of qualitative and quantitative integrated integration is used to guide the intelligent management of satellite Internet content.
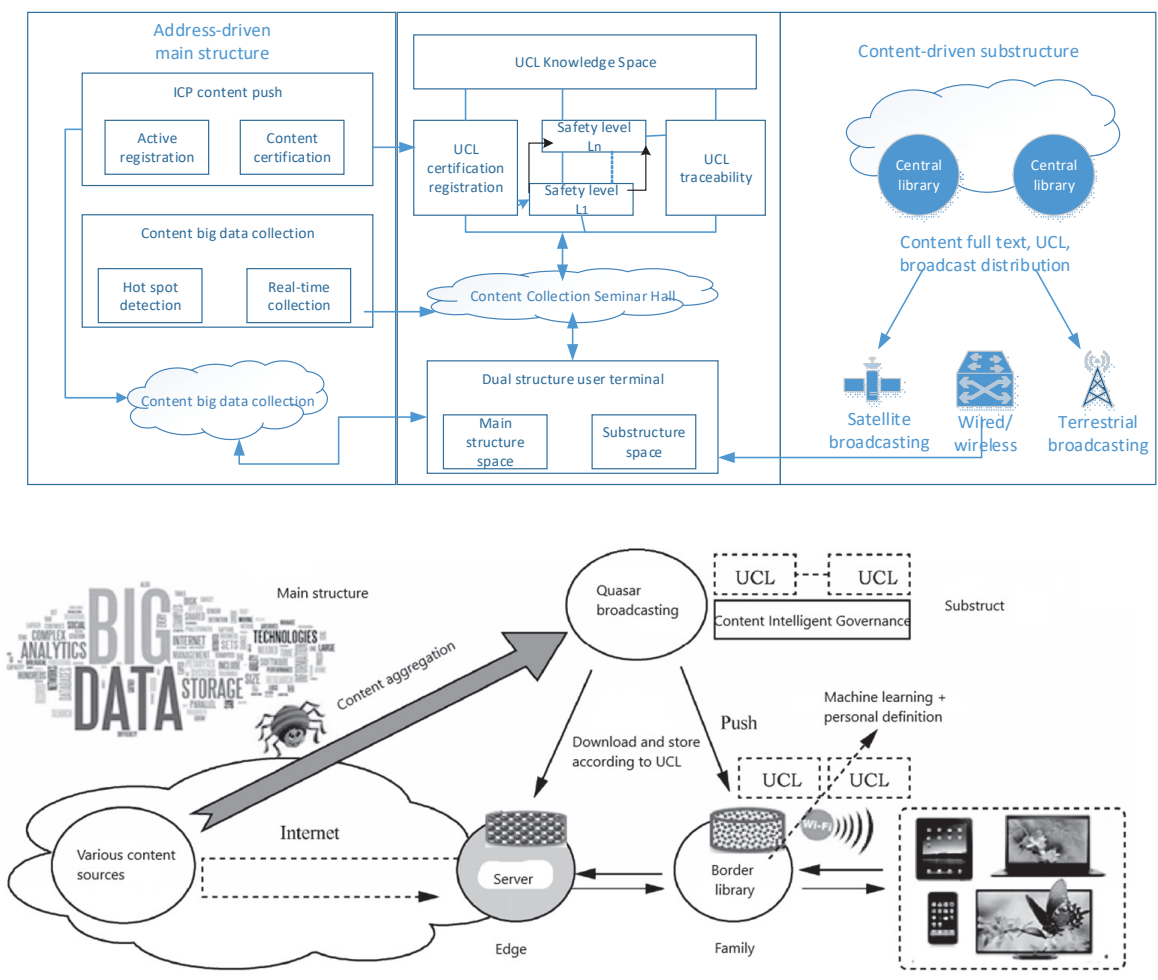

Figure 1 Dual-structure internet architecture reference model.

\subsection{Meta Expression and Construction of UCL}

Matter element, event element, and relation element are generally called meta (Basic-Element). Meta is represented by 3 -tuples of \{object, feature, value $\}$, which constitute the logical cell that describes the problem [9]. 
Meta is usually represented by 3-tuples

$$
B=(N, C, V)=\left[\begin{array}{lll}
\text { Object, } & c_{1}, & v_{1} \\
& c_{2}, & v_{2} \\
& \vdots & \vdots \\
& c_{n}, & v_{n}
\end{array}\right]
$$

Among them, $O$ (Object) represents an object (object, action or relational word), $c_{1}, c_{2}, \ldots, c_{n}$ represent $n$ features of object $O, v_{1}, v_{2}, \ldots, v_{n}$ represent the corresponding values of object $O$ with respect to the above features.

\subsection{Binding of Time and Space}

Cyberspace is facing various security threats and challenges, the root of which lies in the basic network architecture and certain deficiencies in the existing technical system. The Internet and the Global Positioning System are two great inventions of mankind in the 20th century [10]. Based on the dual structure network and the Uniform Content Label UCL national standard, through the integration of these two inventions, the integration of time and space is realized, creating mathematics and physics. The dual-selfconsistent cyberspace endogenous security environment is expected to bring a breakthrough in principle for cracking cyber security challenges [11].

One of the main reasons for the long-term security problems of the network space is that its basic network architecture (TCP/IP protocol architecture) and the existing technology system are difficult to support the close binding of virtual space and real physical space [12]. Although the Internet is an address-driven network, the IP address is essentially a virtual address and is easy to modify and fake. In addition, it uses a destination addressbased forwarding method and does not verify the source address, so it is difficult to trace the source and accountability [13]. It is this technical defect that has deepened the virtuality and uncontrollability of the Internet, making cyberspace a breeding ground for various cyber crimes.

We advocate adding a secondary structure based on the radiation-copy paradigm to the Internet to form a dual-architecture network with conjugate and complementary primary and secondary structures [14]. On this basis, the Uniform Content Label UCL (Uniform Content Label) is a new architecture primitive that binds the two great human inventions of ground-based Internet and space-based global satellite positioning, and realizes self-consistent and non-contradiction through the integration of time and space, and brings endogenous security mechanism to the development of cyberspace. 
Literature [4] found that a complex network that satisfies certain conditions not only has the nature of "small world", but also has "ultra-small world". For the Internet with $N$ users, when the $\lambda$ of the scale-free network meets certain conditions, the average number of hops $D$ is much smaller than the diameter $(D \sim \ln N)$ of the conventional random network and the scalefree network, which satisfies $D \sim \ln \ln N$. When $N$ is 7 billion, $\ln \ln N$ is about 3 . This reveals an ultra-small world that is more closely assembled in space for human society [15]. For the integrated dual-architecture network of heaven and earth, as long as we make full use of satellite network resources, connecting 7 billion people around the world only needs two jump connections (one jump to the sky and one jump to the ground). Not only is the average distance $D$ the smallest, but it has nothing to do with the network node size $N$. Even if the node size exceeds 7 billion, there is no need to increase the average number of hops. This wider, faster, and easierto-achieve spatial aggregation not only enables scale-free "ultra-small world" networks, but also lays the foundation for an unlimited number of nodes in the ubiquitous Internet of Things.

Using ultra-high-precision global satellite uniform time service (such as Beidou, GPS, etc.) as the time stamp can establish the unique integration principle of time and space that reflects the self-consistent time and space [16]. Any data in cyberspace, as long as the time stamp and location that generated the data are bundled, it is no longer an ordinary modulus, but evolves into a vectorized unique label, which no one has the power to fake and tamper with. Endogenous security primitives. The causality of uniqueness can be regarded as the final result of entropy reduction, which has the scientific charm of "unbelievable". Uniqueness ends disagreements and disputes, and consensus begins. The integration of time and space through hash calculation not only protects privacy, but also protects rights and interests. It is a credible token between the physical space and the data space. The time-space-labeled data, or its hash value, is called the trusted element or the trusted root. The "big data" in cyberspace is supposed to be the accumulation of credible elements. After marking the spatio-temporal information, the risk of artificial entropy can be avoided.

Existing blockchain systems are basically built in the asynchronous Internet environment with uncertain delays. Therefore, there are "Triple Paradox" constituted by decentralization, security, and scalability [17]. If you use the dual self-consistent principle of mathematics and physics of space-time aggregation, change the "timing" to "time stamp" on the one hand, and 
change the "unicast" to "broadcast" on the other hand, it may help the blockchain to break through the "triple paradox' difficulties.

This article proposes to add to the Internet a broadcast storage and secondary structure based on the radiation-copy paradigm, forming a dual structure network with conjugate and complementary primary and secondary structures, and binding the Internet and global satellite positioning through the Uniform Content Label UCL to these two great inventions. The development of cyberspace brings new opportunities for endogenous security. The integration of the time and space of the two inventions must be tested by global scientific experiments [18]. The main object of inspection is the application's pervasiveness and ubiquitousness. Because the world and earth channels are physically independent, decoupled, and work in their own ways, the dual-architectured network can integrate the endogenous safety mechanism into all the problems of the network and solve the security problems in the space.

\section{Results on TSN}

\subsection{Time-Sensitive Networking for Satellite}

The satellite Internet is a Time-Sensitive Networking (TSN). The more uniform growth mode can ensure the stability of network delay and reduce the delay jitter. We ran the same replay analyses after scrambling the Uniform Content Labels (UCL) of the satellites during estimation of the template representations. Timeline of satellite transmissions for each label (label ID is the TSN-UCL-assigned transmitter number) [19].

The interval arrival delay of packets is a random variable with normal distribution in the process of network transmission. Different network congestion leads to different normal distribution (different mean and standard deviation) of interval arrival delay. The network topology depends on the physical distances of the satellites [20].

Space information network has become one of the researching field of aeronautics and astronautics. With the development of space information technology and the increase of space communication requirements, new contained services represented by time-sensitive traffic are emerging [21]. For contend service demand of the time sensitive traffic, we proposes a technical architecture for time sensitive space information network. Based on this architecture, its key technologies from three aspects including service, network and resource have been analyzed. 


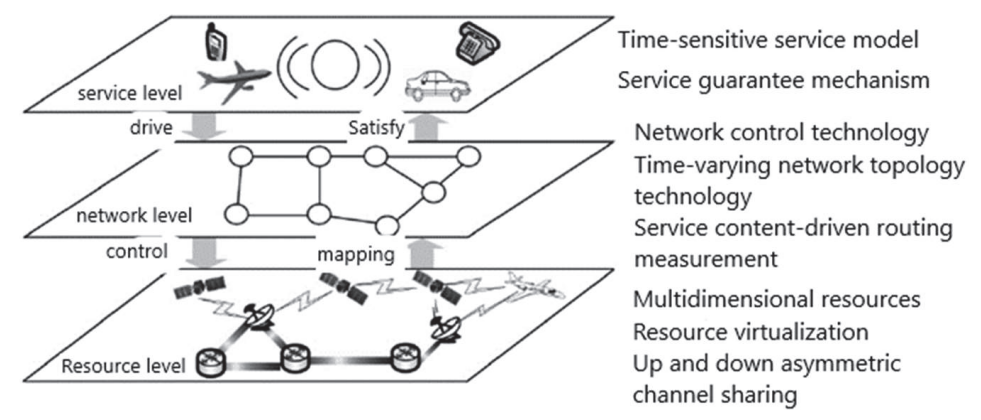

Figure 2 Time-sensitive spatial information network technology architecture.

In order to realize a time-sensitive spatial information network, the existing spatial information network is explored and promoted from three perspectives: business level, network level and resource level, as shown in Figure 2. At the business level, a time-sensitive business model is established, business characteristics are analyzed, and corresponding business guarantee mechanisms are proposed according to business characteristics, and new business requirements are proposed for spatial information networks, identified by UCL, and used as a drive for new network technologies [22]. The network layer, driven by the business layer, establishes a highly adapted network control architecture, combines the characteristics of spatial information network topology changes and the business requirements of time-sensitive services, and conducts research on time-varying network topology technology and routing technology in this scenario, to meet the needs of the business layer. The resource layer mines and analyzes multidimensional spatial information network resources, integrates all available network resources, forms a dual-architecture network of services and routing, and shares resources such as uplink and downlink asymmetric channel sharing, which improves the utilization of resources, and finally integrates multi-dimensional resources [23]. The sharable resources are mapped to the network level through network virtualization technology, supporting network level control technologies and strategies, and realizing effective control of resources at the network level [24].

\subsection{Simulation Environment Establishment}

\subsubsection{The route algorithm for UCL}

UCL can make up for the lack of semantics and management of URL, effectively support the efficient aggregation and ubiquitous distribution of 
content big data, provide personalized active services, and assist in content copyright management.

This algorithm uses UCL in the routing algorithm in the delay-sensitive multilayer satellite network. Based on the SDN solution with cache routing in the network, the logical address method is based on the location of the ground station corresponding to the area where the LEO satellite is located, and the address identifier is assigned to the ground station. The address identifier will be used for routing during data transmission. There are two situations for data packet routing, one is that the content requested by the user already exists in the cache in the network, and the other is that there is no content requested by the user in the cache in the network. The routing algorithm is based on the data processing flow of the $\mathrm{P} 4$ switch. According to a number of specific QoS constraints, the ant colony algorithm is used to select the optimal path that meets the needs.

The satellite nodes involved in the path planning are all LEO satellites. The satellite network is abstracted into a graph. The satellite $v_{i} \in V$ is a vertex, $e(i, j) \in E$ is an edge, and the path from the source $s$ to the destination node $d$ is $P$. The business flow has 3 constraints on the QoS measurement parameters, which are the packet loss rate, the remaining bandwidth of the link, and the transmission success rate, which are represented by $c_{n}$. In addition, the delay is selected as the link cost, and the delay of each link is $t(i, j)$ for TSN. Hence the optimum problem can be presented as,

$$
\begin{cases}\min & t(P) \triangleq \sum_{e(i, j) \in P} t(i, j) \\ \text { s.t. } & \left.e_{1}(P) \triangleq \prod_{e(i, j) \in P} e_{1}(i, j) \leq c_{1}\right\} \\ & e_{2}(P) \triangleq \min e_{2}(i, j) \geq c_{2}, e(i, j) \in P \\ & e_{3}(P) \triangleq \prod_{e(i, j) \in P} e_{3}(i, j) \leq c_{3}\end{cases}
$$

Among them, $t(P)$ represents the transmission delay of the path $\mathrm{P}$ from the source node to the destination node; $c_{1}$ is the upper limit of the packet loss rate on the selected path $\mathrm{P} ; c_{2}$ is the lower limit of the remaining bandwidth on the selected path $\mathrm{P} ; c_{3}$ is the selected path user of the upper limit of the transmission success rate. 
Several parameters of the ant colony algorithm and their meanings are: $\tau_{i, j}(t)$ is the pheromone concentration on the path $(i, j)$ at time $t ; p_{i j}^{k}(t)$ is the probability of ant $k$ from node $i$ to node $j$ at time $t, \rho$ is the pheromone volatilization coefficient, $\alpha$ is the pheromone factor, $\beta$ is the heuristic factor, and $\eta_{i j}$ is the visibility factor. Using $J_{k}(i)$ represents the set of nodes allowed to be selected in the next step.

The steps of ant colony algorithm to solve the multi-constrained optimal path are as follows:

(1) Initialize the relevant parameters first, and set the pheromone amount of each edge at the initial moment to be equal, which is $\tau_{i j}(0)=C$, and $C$ is a constant.

(2) Place the ants at the initial node to start, calculate the transition probability for each ant $k$ and determine its next visit node, until all the ants reach the destination node. Then calculate the total length of each ant's crawling path, record the optimal solution in the current iteration number, that is $L_{k}$, the shortest path $P$, and update the pheromone concentration on the path.

The update rule of pheromone concentration is described as follows:

$$
\tau_{i j}(t+1)= \begin{cases}(1-\rho) \tau_{i j}(t), & e(i, j) \notin p \\ (1-\rho) \tau_{i j}(t)+\rho \cdot F, & e(i, j) \in p\end{cases}
$$

Among them, $F$ is expressed as:

$$
F=-\xi_{1} \cdot F_{1}+\xi_{2} \cdot F_{2}+\xi_{3} \cdot F_{3}+\xi_{4} \cdot F_{4}
$$

In the formula, $\xi_{1}, \xi_{2}, \xi_{3}, \xi_{4}$ are 4 positive real numbers, which respectively represent the relative weight of delay limit, packet loss rate limit, remaining bandwidth limit, and transmission success rate limit. $F_{1}$ means path $p$ delay; $F_{2}$ means when the path is full, the packet loss rate limit is sufficient; $F_{3}$ indicates the remaining bandwidth limit satisfied by the path; $F_{4}$ indicates the transmission success rate limit satisfied by the path. The expressions are as follows:

$$
\begin{aligned}
& F_{1}=\sum_{e(i, j) \in p} t(i, j) \\
& F_{2}= \begin{cases}c_{1}-\sum_{e(i, j) \in p} t(i, j), & c_{1} \geq e_{1}(p) \\
0, & c_{1}<e_{1}(p)\end{cases}
\end{aligned}
$$




$$
\begin{aligned}
& F_{3}= \begin{cases}\min e_{2}(i, j)-c_{2}, e(i, j) \in p \& & c_{2} \leq e_{2}(p) \\
0, & c_{2}>e_{2}(p)\end{cases} \\
& F_{4}= \begin{cases}\prod_{e(i, j) \in p} e_{3}(i, j)-c_{3}, & c_{3} \leq e_{3}(p) \\
0, & c_{3}<e_{3}(p)\end{cases}
\end{aligned}
$$

It can be seen from the above expression that when the selected path has a shorter delay while meeting the delay limit, packet loss rate limit, remaining bandwidth limit, and transmission success rate limit, the pheromone concentration on the path increases, which inspires more ants to choose the path.

(3) After the update of the pheromone concentration is completed, the next iteration is continued, and step (2) is repeated until the predetermined number of iterations is reached or stagnation occurs, the algorithm ends, and the current optimal path is output.

The final output path is the global optimal transmission path obtained according to the current network status information and the QoS requirements of the service.

\subsubsection{Experimental tools}

The experiment uses STK11.01 tool to carry on the relevant simulation of the satellite orbit, and uses the Matlab2016b tool to simulate the algorithm and analyze the experimental results.

\subsubsection{Topology selection}

The experimental topology is adjusted by IRIDIUM, as shown in Table 1. In this way, the number of time slices can be less than 644, the shortest duration is greater than $8 \mathrm{~s}$, and the average duration is greater than $133.17 \mathrm{~s}$, leaving enough time for control domain planning.

Table 1 Satellite orbit parameter setting

\begin{tabular}{lc}
\hline Parameter Name & Value \\
\hline Height/km & 893.8 \\
Inclination angle/ $\left./{ }^{\circ}\right)$ & 86.4 \\
Orbital period/h & $12 / 7$ \\
Number of satellites 66 & 66 \\
Number of tracks 6 & 6 \\
The number of links in the orbit of each satellite is fixed 2 & 2 \\
The number of inter-orbit links for each satellite Dynamic & $0 \sim 2$ \\
\hline
\end{tabular}




\subsubsection{Simulation parameter setting}

The simulation parameters in this paper are set as follows: Differentiated select the value of the LEO flow request rate $\lambda(t)_{n}$, and set the value range to $100 \sim 500 \mathrm{kB} / \mathrm{s}$ to simulate the characteristics of the satellite network control flow. The processing capacity of the slave controller is $8 \mathrm{MB}$. The redundancy factor of the slave controller is set to 0.9 , the average rate of polling LEO is $v_{R}=10 \mathrm{kB} / \mathrm{s}$, and the propagation rate for state synchronization is $v_{S}=1 \mathrm{kB} / \mathrm{s}$.

\subsection{Simulation Result Analysis}

\subsubsection{Algorithm operation time}

In order to shorten the calculation time of the simulated annealing algorithm to adapt to changes in the satellite network topology, the multi-domain control algorithm has designed an iterative jump-out mechanism to shorten the running time of the algorithm. The characteristic of the simulated annealing algorithm is that it can accept poor solutions according to probability and avoid falling into the local optimum. Therefore, a counter is set to record the number of times the new solution is rejected. If it is rejected for consecutive times, this iteration will end early. The relationship between and the value of the objective function and the calculation time of the algorithm is shown in Figure 1. The optimal value of 50 repeated experiments under the same conditions is taken as the final result. The red dotted line in the figure is the target limit value when the iterative jump-out mechanism is not set, and the calculation time at this time is $10.27 \mathrm{~s}$.

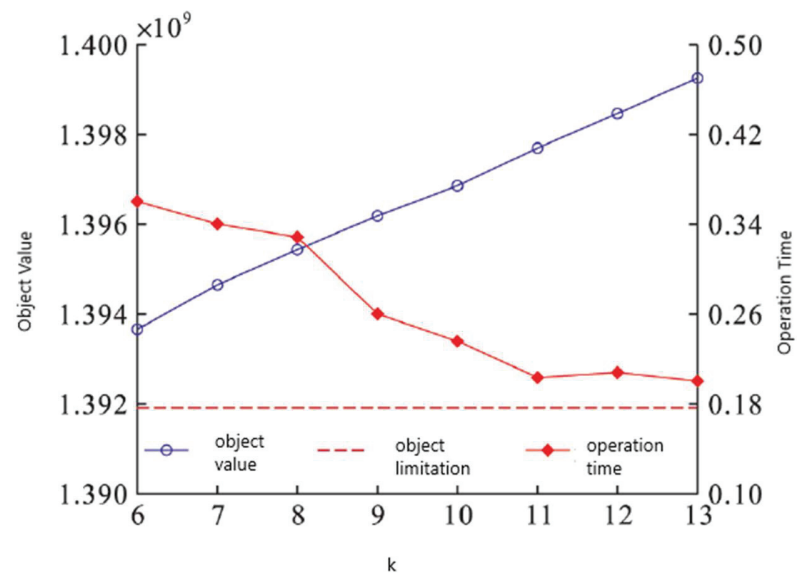

Figure 3 The relationship between object value, operation time and $k$. 
It can be seen from Figure 3 that when $k=11$, the algorithm operation time can be shortened as much as possible while ensuring a good solution result. At this time, the cumulative distribution function of the algorithm operation time is shown in Figure 4. It can be seen that the operation time is basically maintained within $0.56 \mathrm{~s}$, which is much smaller than the shortest duration of the time slice. The iterative jump out mechanism can significantly speed up the calculation speed of the algorithm, so that the algorithm can adapt to the rapid dynamic changes of the satellite network topology.

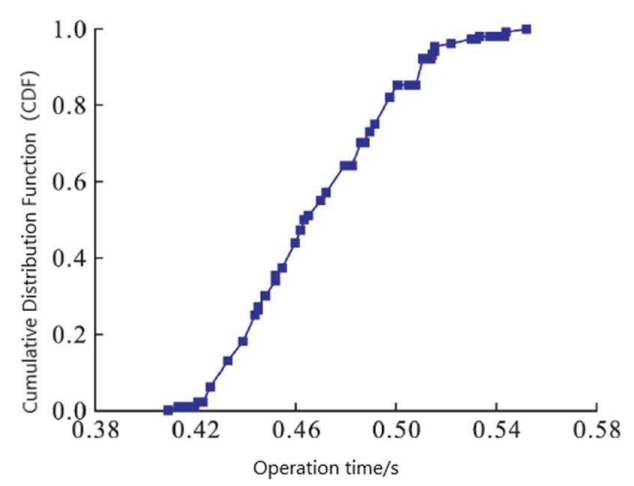

Figure 4 Algorithm operation time.

\subsubsection{Average delay comparison}

It shows the comparison of the route discovery delay between different algorithms. It can be seen from the figure that the system delay is significantly reduced after the UCL label algorithm.

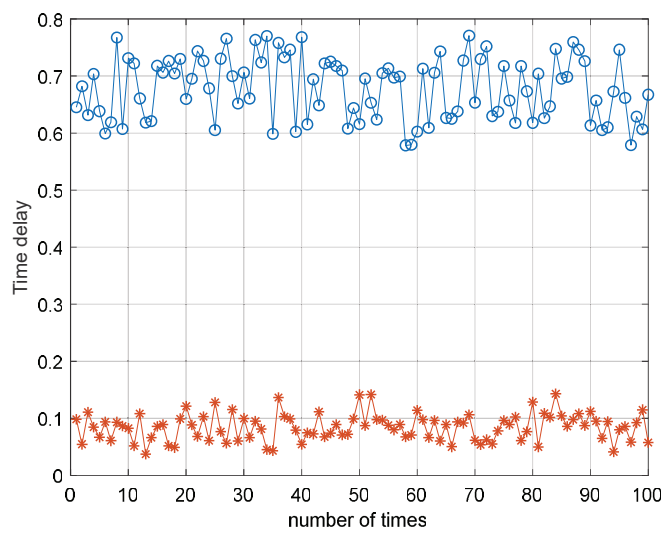

Figure 5 Algorithm operation time. 
Figure 5 shows the comparison of the routing discovery delay between different algorithms. It can be seen from the figure that when the UCL dualarchitecture routing algorithm is used, the delay is significantly reduced.

Through statistical calculation, the average delay of frames in the network is $0.672 \mathrm{~s}$, and the average delay is $0.066 \mathrm{~s}$ by adopt the IP over UCL algorithm.

\subsubsection{Performance analysis of improved algorithms for low-orbit satellite loT}

For the mass access system of the low-orbit satellite Internet of Things, the probability of each user terminal sending data at the same time is very small, most user terminals are in a deep sleep state, and the number of terminals is very large. In actual scenarios, the throughput of the IRSA protocol can reach about 0.8 , which is $53 \%$ higher than the CRDSA protocol and $18 \%$ higher than the CRDSA3 protocol. In comparison, the IRSA protocol is a better choice in the low-orbit satellite IoT access system. We try to take the IRSA protocol as an example. The distribution of data packet copies is $\Lambda(x)=0.5 x^{2}+0.28 x^{3}+0.22 x^{8}$, and the sending probability is added to the user terminal for the next simulation. Suppose the probability that each user terminal has data to send at a certain moment is $p$, the total number of user terminals is $\mathrm{m}$, and the number of time slots in a complete frame is 100. Since the randomness of data transmission for each user terminal is relatively large, that is, at a certain moment, in a large number of user terminals, the distribution of the number of terminals sending data packets is relatively scattered, so assuming the number of terminals sending data at a certain time, Satisfy the normal distribution. According to the nature of the normal distribution and the characteristics of the low-orbit satellite IoT, the mean value of the normal distribution is set to $\mu=p \cdot n$, and the variance is $\delta^{2}=\left(\frac{1}{6} \mu\right)^{2}$. Figures 6 and 7 show the relationship between the throughput of the IRSA protocol and the number of terminals and the relationship between the packet loss rate and the number of terminals when $p=0.5 \%, 1 \%$, and $2 \%$, respectively.

The simulation results show that: as can be seen from Figure 6, after adding the sending probability, increasing the number of terminals and the randomness of the terminal sending data packets, the maximum throughput of the IRSA protocol reaches about 0.63 , which is higher than that of the CRDSA3 protocol in the terminal. The maximum throughput after the transmission probability is higher by about 0.05 , and when the terminal transmission probability is the same and the throughput is the same, the system 


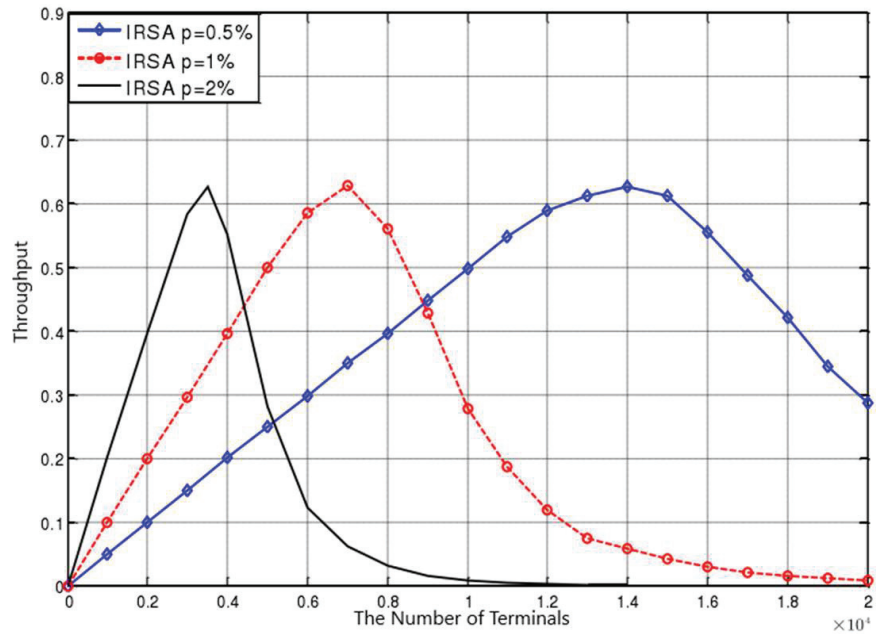

Figure 6 Graph of the relationship between the throughput of the IRSA protocol and the number of terminals.

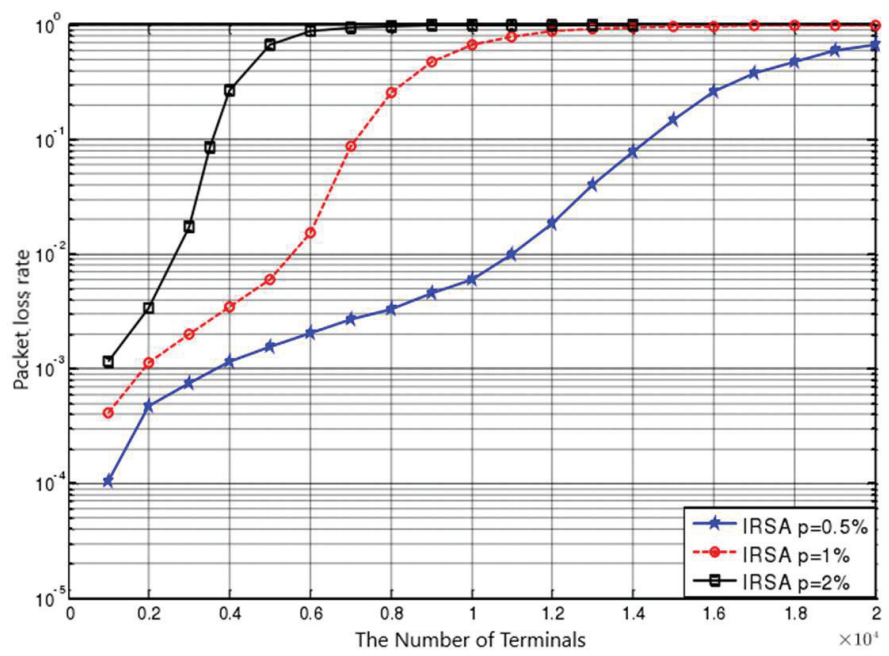

Figure 7 Graph of the relationship between the packet loss rate of the IRSA protocol and the number of terminals.

corresponding to the IRSA protocol has more terminals than the CRDSA protocol. When the throughput is greater than 0.5 , the number of terminals corresponding to different transmission probabilities is also different. When $p=0.5 \%, 1 \%, 2 \%$, the corresponding maximum number of terminals are 
16800,8500 and 4200 respectively. It can be seen from Figure 7 that when $p=0.5 \%$, the packet loss rate is significantly lower than the cases of $p=1 \%$ and $2 \%$; in addition, under the same packet loss rate, $p=0.5 \%$ can be accessed the number of terminals is 2 to 5 times $p=1 \%$ and $2 \%$. When the terminal sending probability is $0.5 \%$ and the allowable packet loss rate is 0.1 , the number of terminals that can be accessed by the IRSA protocol is 14,200 , and the number of terminals that can be accessed by the CRDSA3 protocol is 13,500 . Then, considering the performance of throughput and packet loss rate comprehensively, the performance of the IRSA protocol with the transmission probability of the terminal is higher than that of the CRDSA3 protocol.

It can be seen that as the number of network nodes increases, the average delay and maximum delay will gradually increase, and the probability of successful frame transmission will gradually decrease.

Compared with the non-adaptive maximum contention window, the average and maximum delay is reduced, while the network throughput, effective throughput and the successful transmission rate of the frame are almost unchanged.

\section{Discussion}

The Internet is already the foundation medium to access, link and use all these data. Satellite communication [25], imagery and remote sensing are quickly entering the mainstream. Our findings demonstrate for the first time that satellite's chirp timing is sensitive to linguistic content. The discussion above has focused on just one form of Internet-mediated research, qualitative analysis of the content of online discussion posted on satellite networks.

There are many reasons why satellite tags stop transmitting including content label malfunction [26]. Our studies focused on all satellite content, and the results were largely driven by mini- or macro-satellites. Meanwhile, Internet or web surveys are less costly and more time efficient but also have limitations especially including coverage error. The variability in the quality of the content label on the Internet has been demonstrated in many studies from the early stages of Internet adoption. Consistent with a previous qualitative study on why people opt to use Internet intervention, it was found that the content of the intervention may influence the motivation of the users of satellite to take part in the webinar. Indeed, satellite Internet's safety is difficult to regulate, including because of access to internationally transmitted content [27]. 
This result shows that satellite-free and satellite-based time stamps are equally sensitive to the delay of this spatial routing. In this way, the satellite spatio-temporal data obtained using unified content will be shared with the knowledge base. Future data coordination plans should also focus on the coordination of content labeling and time-sensitive frameworks.

UCLs, Uniform Content Label, are the base concept on which the semantic web technologies were developed, as well as the satellite gateway machine was used to provide Internet access and inter-satellite routing and switching functionality to the network.

\section{Acknowledge}

The author thanked Academician Li Youping and Prof. Yang Peng for their academic discussions, and referred to UCL standards and papers.

\section{References}

[1] Yang Peng, Li Youping. Dual-structure Internet that supports content intelligent governance[J]. Journal on Communications, 2019, 040(009):1-14.

[2] Wang Jingli, Li Xingsen. Elementary expression and construction strategy of Uniform Content Label [J]. Journal of Guangdong University of Technology, 2014(02): 14-20.

[3] Li Youping, Cheng Guang, Yang Peng. Space-time assembly creates endogenous security in cyberspace[J]. Cyberspace Security, 2020, v.11; No.126(08):5-9.

[4] Cohen R., Havlin S. Scale-Free Networks Are Ultrasmall[J]. Physical Review Letters, 2003, 90(5): 058701.

[5] Yang Hui, Bai Wei, Zhang Jie. Research on key technologies of time-sensitive spatial information network[J]. Radio Communication Technology, 2017, 43(003): 8-12.

[6] Yang Peng, Li Youping. Universal model and realization mode of broadcast network architecture[J]. Chinese Journal of Electronics, 2015(5).

[7] Yang Peng, Zhang Xiaogang, Li Youping, etc. A video recommendation method and device based on UCL semantic indexing.

[8] Ji Yeli. Research on SDN-based routing scheme for the nearest distribution network of content. Beijing University of Posts and Telecommunications, 2019. 
[9] Zhang Chi, Han Li, Yang Hong. Key technologies and standardization status of time-sensitive networks[J]. Automation Instrumentation, 2020, v.41; No.463(03):100-104.

[10] Zhang Han, Huang Xiangyue, Meng Xiangjun, et al. Research on SDN/NFV-based world-ground integration network architecture[J]. Military Communication Technology, 2017(02): 35-40.

[11] Yin Zhizhong, Zhang Jiguang, Zhou Xianwei, et al. Low-latency multilayer satellite network based on multi-region division[J]. Telecommunications Technology, 2010, 50(002): 78-83.

[12] Sheng Min, Li Jiandong, Shi Yan. Delay-sensitive adaptive routing protocol applied to Ad Hoc network[J]. High Technology Communications, 2003(06):1-4.

[13] Sun Xuehai. The influence of satellite communication delay on information network [J]. Shanxi Electronic Technology, 1999, 000(001): 8-9.

[14] Wang He. Research on performance optimization technology of satellite network transmission protocol [D]. Harbin Institute of Technology, 2011.

[15] Ma Huan. Efficient transmission of delay-sensitive services in autonomous wireless networks [D]. Xidian University, 2013.

[16] Hou Y, Diao Y. A uniform content label push method based on DRM broadcasting[C]. International Conference on Systems \& Informatics. IEEE, 2017.

[17] Liu Zhe. Exploration of exporting files from highly sensitive network to low sensitive network[J]. Computer Engineering and Design, 2018, 039(001):55-60.

[18] Ma Huan. Efficient transmission of delay-sensitive services in autonomous wireless networks [D]. Xidian University.

[19] Liu Chang, Shi Huipeng, Li Wei. Analysis of International Coordination Between Satellite Networks [C]// Proceedings of the 2014 National Wireless and Mobile Communication Conference. 2014.

[20] Cong Peizhuang, Tian Ye, Gong Xiangyang, et al. Overview of key protocols and application scenarios of time-sensitive networks[J]. Telecommunications Science, 2019, v.35(10):37-48.

[21] Wang Shuo. Time-sensitive network technology and its application in the Industrial Internet [J]. Electronic Technology and Software Engineering, 2018, No.143(21):34+115. 
[22] Miao Jiansong, Wang Pengjie, Yang Zeyun. Satellite Internet text sensitive information detection method and device based on deep learning:, CN111078879A[P]. 2020.

[23] Yuan Linfeng, Cheng Wenqing, Du Xu, et al. Sensor network routing protocol based on restricted equivalent delay [J]. Small Microcomputer System, 2007, 28(009):1549-1553.

[24] Ru Xulong. Research on Time Synchronization Technology in Time Sensitive Network [D]. Xidian University.

[25] Peng Li, Jiang Mingyan. Anti-delay sensitivity cross-layer adaptive resource allocation scheme $[\mathrm{J}]$. Application Research of Computers, 2010(03):1122-1125.

[26] Guo Xiong, Yang Hong, Li Mengliang. Edge computing and time-sensitive network integration technology research and standard progress[J]. China New Telecommunications, 2020, v.22(05):63-65.

[27] Song Huazhen. Overview of time-sensitive network technology [J]. Automation Instrumentation, 2020, 041(002):1-9.

\section{Biographies}

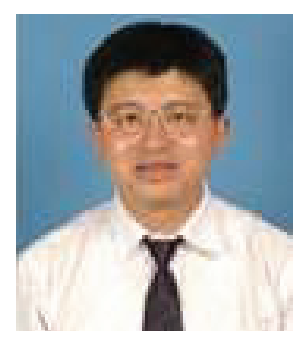

Jie Shen, obtained a bachelor's degree from Shanghai Jiao tong University and a master's and doctorate in electronics from Beijing University of Posts and Telecommunications, Beijing, P.R.C. He obtained the title of communications engineer in 2004, and obtained the title of senior engineer in communications in 2009. In 2014, he was awarded the title of professor-level senior engineer in electronics respectively. His research interests include: wireless and optical communications, data communications, quantum information, and microelectronics. 


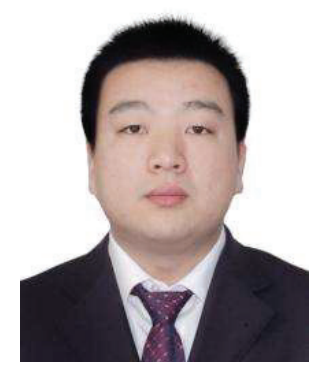

Wenqi Dong, obtained a Master degree from Beijing University of Chemical Technology, and obtained his second Master degree from North China Electric Power University,Senior Engineer of State Grid Jibei Zhangjiakou Wind and Solar Energy Storage Storage and Transportation New Energy Co. Ltd. The main research directions are: Multi-type energy storage technology, Energy Internet technology, wireless and optical communications.

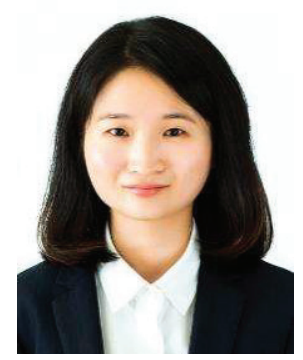

Jing Wang received her B.Sc. degree in Electrical Engineering from Hebei Normal University of Science \& Technology; M.Sc. degree in Business Administration from North China Electric Power University.

Jing Wang has key positions in the field of information and communication at State Grid Jibei Zhangjiakou Wind and Solar Energy Storage and Transportation New Energy Co., Ltd since 2012. As a Telecommunication expert, she has rich experience in the research and management of new energy communication technology. She is committed to researching the wireless communication technology in power control and acquisition services, in order to promote the upgrade of power grid to energy Internet and build a new power system supported by IoT Communication and digital technology. 


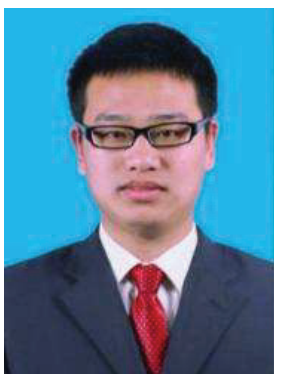

Peng Zang, obtained a bachelor's degree from China University of Mining and Technology, pursuing a master's degree in electrical engineering at China Agricultural University. The main research directions are: wind-solar storage operation control technology.

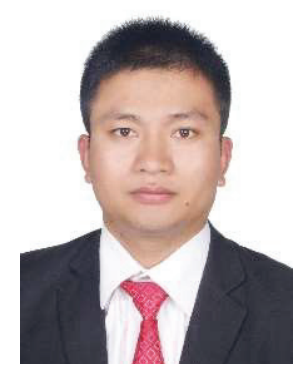

Xuewei Shi, Bachelor of Hebei University of Science \& Technology, New energy testing technical engineer of State Grid Jibei Zhangjiakou Power Wind and Solar Storage Company. His main research directions are: new energy power generation technology, large-scale energy storage operation and maintenance technology. 


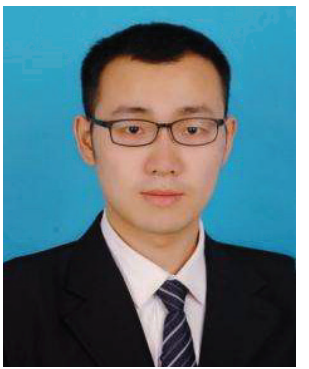

Yang Wang, obtain Master from North China Electric Power University, Engineer of State Grid Jibei Zhangjiakou Wind and Solar Energy Storage and Transportation New Energy Co. Ltd. The main research directions are: wind-solar storage and transportation demonstration project and economic analysis. 